\title{
CRISPR Cas9 Tip of an iceberg: It's potential in genome editing and many more.
}

\author{
Jalal Ahmad $^{1}$, Nayyer Siddique ${ }^{2}$ \\ ${ }^{1}$ Undergraduate student at Center of Microbiology and Biotechnology, University of Peshawar, \\ Pakistan \\ Emails: std100609@uop.edu.pk,imjala197@gmail.com \\ ${ }^{2}$ Graduate student at Institute of Basic Medical Sciences, Khyber Medical University, Peshawar, \\ Pakistan \\ Emails: nayyersiddique12@gmail.com
}

\begin{abstract}
:
Clustered regularly interspaced short palindromic repeats or CRISPR, one of the major technological tools from nature's toolbox, has revolutionized the scientific world with its potential use in humans and plants. CRISPR Cas9 was first known as an adaptive immune system of bacteria. It is a system that cleaves foreign DNA. It has been exploited to be used as a genome editing tool for correcting genetic diseases in humans, for plants to create stress-resistant plants, and for a variety of different purposes. This review provides a basic overview of its applications in different areas of biological research. It has immense potential for a variety of researches, but it's still a mystery for science. It feels like scientists just know a tip of an iceberg.
\end{abstract}

Keywords: CRISPR-Cas9, Genome editing, plant editing, bacterial immune system, genetic disease.

\section{Introduction:}

A drop in the ocean of technological advancement: CRISPR Cas9. CRISPR abbreviated as clustered regularly interspaced short palindromic repeats were first identified in bacterial species E.coli [1]. These repeated sequences of DNA were then detected in different species of bacteria and algae [2]. There Cas (CRISPR associated) genes were identified in 2002 and after that different scientist paved the way to an understanding that these sequences have extrachromosomal and phage-associated origins [3-5]. Later on, research went on, on the origin and function of these repeats, where a team of scientists identified CRISPR as the adaptive immune system of the bacterial and algae species [6,7]. It was shown that mature CRISPR RNAs serve as a guide for the CRISPR protein to act on the complementary sequences[8], it was also shown that the transcribed sequence of the CRISPR loci serves as a code for protein which has helicase and nuclease domains $[6,7,9]$.

In 2012, pioneering biochemists Jennifer Doudna and Emanuelle Charpentier revealed that cas9 can be guided manually to cleave a target DNA in-vitro [10]. This giant leap in the field of genome engineering opened doors for multiple types of research. Scientists used CRISPR Cas9 
for engineering genomes of mammals [11, 12]. Other organisms have also been treated with CRISPR Cas9 see references [13-17].

Furthermore, CRISPR Cas9 has also the potential to meet the need of the incoming food shortage disaster as the world population is getting higher. For this purpose, a scientist has exploited the role of CRISPR Cas9 in plant genome editing to produce high-yielding plants, drought resistant plants, and disease resistant plants $[18,19]$. In this review, we have highlighted its revolutionizing potential in different areas of research. We have discussed briefly the applications of Cas9 in genome engineering in human, plants and animals. Apart from genome editing CRISPR Cas9 system has also been exploited for several different purposes including genome imaging, genome screening, and regulating genome expression, reviewed in the next section.

Although CRISPR Cas9's ability is no secret, its potential in genome engineering in plants and animals has revolutionized the science of biotechnology, but still, after 9 years we find ourselves ignorant of its true potential, we believe we still know just a drop in an ocean of nature's toolbox.

\section{CRISPR Cas9 functionality and biology:}

Like all the other technologies, CRISPR Cas9 technology also has a proposed working model. In prokaryotic organisms Cas9 technology works in a three-way fashion i) the identification of the pathogenic or unidentified DNA entrance into the cell, ii) the maturation of the crRNA, iii) the direct cleavage of the unidentified DNA sequence with the help of the sequence-specific Cas enzymes. The activity of the Cas9 protein is maintained by two types of RNAs. The crRNA (CRISPR RNA) and the trans-activating (tracrRNA) [20, 21]. When pathogenic DNA enters the cell. The pre-crRNA is transcribed of the CRISPR array. The pre-crRNA is then cleaved into crRNA by tracrRNA, this tracrRNA is helpful for cas9 binding, cas9 mediated target cleavage, and crRNA processing $[10,22]$. The tracrRNA helps in the cas9 and thus produces a mature crRNA. The complex of crRNA, tracrRNA, and cas9 seeks out and finds the complementary sequence to crRNA to introduced a DSB [20,21]. In 2011, pioneering biochemists Charpentier and Doudna combine the crRNA:tracrRNA complex into single guide RNA, which can be complexed into Cas9 and used to cleave the target DNA [10].

Cas9 structure consists of two main components, the NUC lobe, and the $\alpha$-helical region. The NUC lobe of the Cas9 contains the HNH nuclease domain, Ruv-C like nuclease domain, a PAM interacting domain, and an evolutionary wedge domain (WED). The HNH nuclease domain and Ruv-C like nuclease domain are used for cleaving the target DNA. The PI domain enhances the specificity of the Cas9 by interacting with the PAM regions. The wedge domain interacts with the backbone of the PAM region [23, 24]. The HNH nuclease domain cleaves the crRNA complementary strand, while the Ruv-c like domain cleaves the non-complementary or the opposite strand [20,21].

\section{CRISPR Cas9 in genome/epigenetic editing, genome screening, and imaging:}

\section{Cas9 based genome/epigenetic editing:}

As new technologies are introduced into the scientific community, the world of scientists explores its potential to edit the human genome for different therapeutic purposes. Such is in the 
case of CRISPR Cas9, as its potential create a double-stranded break (DSB) in the genome precisely with the help of CRISPR RNA or guide RNA [10]. Here we will review some examples/studies of genome editing carried out with the help of CRISPR Cas9. Usage of Cas9 technology makes it easier for scientists to perform their desired edits that were previously difficult to attain with transcriptional-activator-like effector nucleases (TALENs) and zinc-finger nucleases (ZFNs) [25, 26].

Many studies have been carried out to correct or cure genetic diseases which include Duchene muscular dystrophy, Fanconi anemia, hemophilia, and beta-thalassemia. In one study, this was regarded as the first successful study to correct genetic disease in post-natal animals in vivo. In this study, hereditary type 1 tyrosinemia was cured in a mouse model. Type 1 tyrosinemia is caused by a deficiency of FAH (fymarylacetoacetate hydrolase). The authors delivered the cas9 and sgRNA along with a DNA template for HDR (homology-directed repair), which resulted in the correction of the mutated gene [27]. In another study which was also carried out in vivo, in this study disruption of gene encoding proprotein convertase subtilisin/Kexin type 9 was performed in mouse liver. The disruption of the target gene showed low-level blood cholesterol [28]. Another Cas9-mediated in vivo gene editing was also applied on mice zygotes that were heterozygotes for dominant-negative for cataract mutation of the CRYGC gene. The resulted therapy helped in acquiring a cataract-free progeny [29]. In 2014 Long, used the same approach in an attempt to cure Duchene muscular dystrophy (DMD) in MDX mice [30]. Anyhow, the correction of disease in post-natal MDX mice has also been reported by delivering sg-RNA along sp-Cas9 or sa-Cas9 with the help of AAV [31-33]. In another, in vivo study, a mouse with homozygous $\mathrm{G}$ to A point mutation in the FAH gene was corrected by delivering ssDNA along with Cas9 and sg-RNA via HDI into an adult mouse. A significant amount of wild-type FAH protein was observed [27]. A genetic mutation in the CFTR gene that happens to cause cystic fibrosis was corrected with the help of Cas9 editing [34]. In an attempt to cure retinitis pigmentosa, a rhodopsin mutation was corrected in rats using suitable sg-RNA to target the mutation via subretinal injection [35]. CRISPR-mediated gene therapy was performed to correct a pathogenic mutation in the MYBPC3 gene, which causes HCM (hypertrophic cardiomyopathy) [36]. In another gene therapy study, LCA type 10 is caused by a mutation in gene CEP290. Cas9 technology was used to cure the mutation via NHEJ (non-homologous end joining) of the desired mutated gene [37]. CRISPR-Cas9 has also been used extensively in immunological disorders. JAK3 mutations which result in a form of T-B + SCID was corrected by a team of a scientist using CRISPR-Cas9 technology via HDR in exon 14 of a mutated copy of JAK3 gene from patient-derived iPSCs [38]. Hyperammonemia results from a deficiency of an OTC enzyme. The authors corrected the point mutations from G to A via dual AAV mediated delivery (one contains the sg RNA and template for HDR while the other contained CRISPR Cas9), of the OTC enzyme gene [39]. In 2018, scientists corrected a $\mathrm{G}$ to A point mutation in the INS gene exon 2 of a PNDM patient-derived iPSCs [40].

Alongside having the potential to correct genetic diseases the CRISPR Cas9 has also some antiviral properties. In 2014, a team of scientists expressed a CCR5 $\Delta 32$ mutation with the help of Cas9 and piggyback technologies to form homozygous iPSCs resistant to HIV-1 [41]. During another study, scientists used adenovirus vectors for delivering Cas 9 for targeting the exon 4 of an open reading frame of the CCR5 gene and disrupted the CCR5 expression through NHEJ, which resulted in HIV-1 resistivity [42]. Cas9 has further been used for targeting human viruses which include herpes[43], papillomaviruses[44-46], and HBV [47]. 
Apart from editing the gene sequences or correcting them, Cas9 can also be used to regulate the expression of genes by its ability of site-specific epigenome editing. For achieving epigenome editing, a variant of the cas9 system called dCas9 is used. dCas9 has a deactivated form of its nuclease activity which can help in editing the epigenome without changing the DNA sequence $[48,49]$. In a study, scientists formed a complex dCas9 and histone demethylase LSD1. They targeted the enhancers of some genes, which were essential for maintaining pluripotency of mouse embryonic cells (mESCs). They showed that dCas9 efficiently lowered the expression of the genes that were controlled by those enhancers [50]. In another study, scientists were able to lower the expression of the globin gene with the dCas9-KRAB fusion [51]. The strategy used by Hilton et al. they formed a complex of dCas9 and the catalytic domain of the histone acetyltransferase p300. Using this method scientists acquired the activation of several endogenous genes by targeting their promoter regions [52].

\section{Cas9 in genomic imaging and screening:}

The sequence specificity of Cas9 and its ease of handling have made it a huge tool for genome editing, but aside from editing the genome of an organism, we have explored its ability in epigenome editing. In this section, we will provide a short overview of Cas9's ability in genomic imaging and screening. Genomic screening and imaging provide insights into finding diseaserelated markers and many other genomic loci, with Cas9 specificity it has become easier.

Genome imaging based on Cas9 has taken advantage of nucleotide interactions and protein-DNA interaction for imaging genomic loci. In an exciting work, this was regarded as the first use of Cas9 for genomic imaging. The authors combined dCas9 with an enhanced GFP to visualize the genomic loci (coding and non-coding sequences) in human cells. In this published work, the repetitive sequences were tracked using a single sgRNA, while the non-repetitive sequences were tracked using multiple sgRNA's [53]. Another group of scientists used the same approach to track down centromeres, telomeres, and pericentric regions in mESCs [54]. Scientists used many approaches to expand the area of genomic imaging using cas 9 technology. A group of scientists fused the dCas9 technology with SunTag peptide to amplify the fluorescent signal transmitted by dCas9 [55]. In another method, dCas9 orthologs were fused with different FPs (fluorescent proteins). These approaches were used to target distinct genomic loci due to their corresponding sgRNAs [56]. CASFISH is a method used for rapid imaging of the genomic sequences and structures by using dCas9 is labeled with a fluorescent protein in fixed cells and tissues assembled in vitro [57].

Genome-wide screening can help in identifying potential drug targets or help in identifying sensitive genes i.e., their regulation patterns in a certain disease, which could be used to design a strategy for that particular disease. Here in this review, we will also outline some of the studies concerning genome screening. In a study published in 2019, the authors described how KEAP1 gene disruption can lead to resistance to sorafenib, lenvatenib, and regorfenib in advanced HCC (hepatocellular carcinoma), by using Model-based Analysis of Genome-wide CRISPR-Cas9 knockout (MAGeCK)[58]. A recent study, with the help of genome-wide screening with the help of CRISPR Cas9, showed MR1 as the recognition molecule by T cell targeting cancer in HLA-independent immunotherapies [59]. A group of scientists identified a group of long noncoding RNA (lncRNAs) screened with help of Cas9 and sgRNAs libraries that were important in the cellular growth of myeloid leukemia K562 cells [60]. Many genome screenings studies have 
taken place since the inception of CRISPR Cas9 sequence-specific ability, which is reviewed in (see ref. [61, 62]).

\section{CRISPR Cas9 in Plant genome editing:}

Tales of Cas9 editing in human genome editing, creating model organisms, and studying or screening human or animal genomes are being circled like a wrecking ball in modern scientific knowledge. CRISPR cas9 has also been used in editing plant genomes to make them more resistant to diseases, to increase yield, or to make them more nutritional. Many studies in the past few years have been published regarding the usage of cas9 technology for editing plant genomes for different purposes. Here we will provide a short overview of some of those studies.

Classical methods to improve crop yield are tedious, time-consuming, and require lots of patience as the perfect product could take almost 7 years to be used. In this regard, CRISPR cas9 has sped up the process of improving the yield. In a study, four rice genes were targeted with cas9 for mutagenesis i.e., IPA1, GS3, DEP1, and Gn1a. The alteration showed increased grain size and number with a mutation rate of $27.5 \%$ to $67 \%$ [63]. Editing of four-grain weight-related genes namely GW2, GW5, GW6, and GS3 showed great improvement in grain weight and size [64]. In addition, the heading gene in rice was mutated with the help of cas9, the edited plant showed early flowering and maturity in comparison to the wild type [65]. Cas9 technology was used to mediate a gene knockout of rice sugar transporter gene OsSWEET11, the result showed reduced sugar content, grain filling, and grain weight. So, this led to the conclusion that overexpression of the studied gene may improve the rice yield [66]. Furthermore, the CRISPR cas9 technology has been used to produce seedless tomatoes. Cas9 mediated mutagenesis in a gene called the SIIAA9 gene resulted in an altered leaf shape and seedless tomatoes [67]. Cas9 directed mutation of the SP4G gene of tomato showed rapid flowering and improved yield [68].

In contrast to improving the yields of plants, CRISPR Cas9 has also been used to improve the nutritional qualities of food. A study showed that targeting 3 fatty acid desaturase genes can improve the fatty content in camelina sativa up to 50\% [69]. Another study showed that frameshift mutations in the SBEIIb gene increased the levels of amylose and starch by $25 \%$ and 9.5\% in rice [70]. In another study, it was shown that targeting RPL and PPR could increase lysine and tryptophan contents by a $12.5 \%$ reduction of zein proteins in maize kernels [71]. Improving a plant's response to disease can also be enhanced with CRISPR cas9 genome editing, which leads to disease-resistant plants. We will provide a short overview of some of the related studies. A cas 9 editing of the effector binding element $\mathrm{EBE}_{\mathrm{pthA} 4}$ in the promoter of the citrus canker susceptibility gene CsLOB1 showed resistance to Xanthomonas ciri and complete knockout of the gene showed enhanced resistance [72]. In another study cas 9 mediated editing of the ethylene response factor, OsERF922, showed improved rice blast symptoms and it leads to better agronomic performances [73]. A study was carried out to produce a non-transgenic tomato variety, which was mildew resistant by editing mildew resistant locus o (SIMlo) [74]. A viral resistant Arabidopsis was produced by editing the eukaryotic translation initiation factor with cas9, which showed complete resistance to the Turnip Mosaic virus, this edit was also passed onto the subsequent generation without any damage to the plant trait [75]. A similar point mutation was carried out in the elF4E gene in cucumber which showed resistant Papaya ringspot mosaic virus-W, Zucchini yellow mosaic virus, and Ipomovirus cucumber vein yellowing virus 
[76]. These mutations in plant genomes could take years to process but with the help of robust, reliable, and simple Cas9 technology all of them can be achieved in one generation. For further study see review: $[18,19]$.

\section{Conclusion:}

Clustered regularly interspaced short palindromic repeats (CRISPR-Cas9), have risen to the level of being an astounding hope for scientists. The usage of CRISPR-Cas9 shows its great importance in different biological fields that include genetic engineering, therapeutics, genomic screening, and its relevant significance in plant biotechnology. As the above studies show its pioneering mechanism in genome editing, CRISPR-Cas9 is a hope for many gene-related diseases like muscular dystrophy, sickle cell anemia, etc. CRISPR-cas9's ability in plant engineering can provide an astounding hope for disease-resistant crops and high-yield crops to overcome the world's nutritional needs. As far as we know we've just discovered a tip of an iceberg, there's yet so much to understand about the Cas9 mechanism and its functioning. We're still very far from its end or what we have to know.

\section{References:}

1. Ishino, Y., et al., Nucleotide sequence of the iap gene, responsible for alkaline phosphatase isozyme conversion in Escherichia coli, and identification of the gene product. Journal of bacteriology, 1987. 169(12): p. 5429.

2. Mojica, F.J., et al., Biological significance of a family of regularly spaced repeats in the genomes of Archaea, Bacteria and mitochondria. Molecular microbiology, 2000. 36(1): p. 244-246.

3. Bolotin, A., et al., Clustered regularly interspaced short palindrome repeats (CRISPRS) have spacers of extrachromosomal origin. Microbiology, 2005. 151(8): p. 2551-2561.

4. Mojica, F.J., J. García-Martínez, and E. Soria, Intervening sequences of regularly spaced prokaryotic repeats derive from foreign genetic elements. Journal of molecular evolution, 2005. 60(2): p. 174-182.

5. Pourcel, C., G. Salvignol, and G. Vergnaud, CRISPR elements in Yersinia pestis acquire new repeats by preferential uptake of bacteriophage DNA, and provide additional tools for evolutionary studies. Microbiology, 2005. 151(3): p. 653-663.

6. Makarova, K.S., et al., A putative RNA-interference-based immune system in prokaryotes: computational analysis of the predicted enzymatic machinery, functional analogies with eukaryotic RNAi, and hypothetical mechanisms of action. Biology direct, 2006. 1(1): p. 1-26.

7. Barrangou, R., et al., CRISPR provides acquired resistance against viruses in prokaryotes. Science, 2007. 315(5819): p. 1709-1712.

8. Garneau, J.E., et al., The CRISPR/Cas bacterial immune system cleaves bacteriophage and plasmid DNA. Nature, 2010. 468(7320): p. 67-71.

9. Karthik, L., et al., Protease inhibitors from marine actinobacteria as a potential source for antimalarial compound. PloS one, 2014. 9(3): p. e90972.

10. Jinek, M., et al., A programmable dual-RNA-guided DNA endonuclease in adaptive bacterial immunity. science, 2012. 337(6096): p. 816-821.

11. Mali, P., et al., RNA-guided human genome engineering via Cas9. Science, 2013. 339(6121): $\mathrm{p}$. 823-826. 
12. Cong, L., et al., Multiplex genome engineering using CRISPR/Cas systems. Science, 2013. 339(6121): p. 819-823.

13. Chang, N., et al., Genome editing with RNA-guided Cas9 nuclease in Zebrafish embryos. Cell Research, 2013. 23(4): p. 465-472.

14. Nakayama, T., et al., Simple and efficient CRISPR/Cas9-mediated targeted mutagenesis in Xenopus tropicalis. genesis, 2013. 51(12): p. 835-843.

15. Li, D., et al., Heritable gene targeting in the mouse and rat using a CRISPR-Cas system. Nature biotechnology, 2013. 31(8): p. 681-683.

16. Li, W., et al., Simultaneous generation and germline transmission of multiple gene mutations in rat using CRISPR-Cas systems. Nature biotechnology, 2013. 31(8): p. 684-686.

17. Hai, T., et al., One-step generation of knockout pigs by zygote injection of CRISPR/Cas system. Cell research, 2014. 24(3): p. 372-375.

18. Haque, E., et al., Application of CRISPR/Cas9 genome editing technology for the improvement of crops cultivated in tropical climates: recent progress, prospects, and challenges. Frontiers in plant science, 2018. 9: p. 617.

19. Hussain, B., S.J. Lucas, and H. Budak, CRISPR/Cas9 in plants: at play in the genome and at work for crop improvement. Briefings in functional genomics, 2018. 17(5): p. 319-328.

20. Doudna, J.A. and E. Charpentier, The new frontier of genome engineering with CRISPR-Cas9. Science, 2014. 346(6213).

21. Hsu, P.D., E.S. Lander, and F. Zhang, Development and applications of CRISPR-Cas9 for genome engineering. Cell, 2014. 157(6): p. 1262-1278.

22. Deltcheva, E., et al., CRISPR RNA maturation by trans-encoded small RNA and host factor RNase III. Nature, 2011. 471(7340): p. 602-607.

23. Nishimasu, H., et al., Crystal structure of Staphylococcus aureus Cas9. Cell, 2015. 162(5): p. 11131126.

24. Jinek, M., et al., Structures of Cas9 endonucleases reveal RNA-mediated conformational activation. Science, 2014. 343(6176).

25. Ding, Q., et al., Enhanced efficiency of human pluripotent stem cell genome editing through replacing TALENs with CRISPRs. Cell stem cell, 2013. 12(4): p. 393.

26. Byrne, S.M., P. Mali, and G.M. Church, Genome editing in human stem cells. Methods in enzymology, 2014. 546: p. 119-138.

27. Yin, H., et al., Genome editing with Cas9 in adult mice corrects a disease mutation and phenotype. Nature biotechnology, 2014. 32(6): p. 551-553.

28. Ding, Q., et al., Permanent alteration of PCSK9 with in vivo CRISPR-Cas9 genome editing. Circulation research, 2014. 115(5): p. 488-492.

29. Wu, Y., et al., Correction of a genetic disease in mouse via use of CRISPR-Cas9. Cell stem cell, 2013. 13(6): p. 659-662.

30. Long, C., et al., Prevention of muscular dystrophy in mice by CRISPR/Cas9-mediated editing of germline DNA. Science, 2014. 345(6201): p. 1184-1188.

31. Long, C., et al., Postnatal genome editing partially restores dystrophin expression in a mouse model of muscular dystrophy. Science, 2016. 351(6271): p. 400-403.

32. Nelson, C.E., et al., In vivo genome editing improves muscle function in a mouse model of Duchenne muscular dystrophy. Science, 2016. 351(6271): p. 403-407.

33. Tabebordbar, M., et al., In vivo gene editing in dystrophic mouse muscle and muscle stem cells. Science, 2016. 351(6271): p. 407-411.

34. Schwank, G., et al., Functional repair of CFTR by CRISPR/Cas9 in intestinal stem cell organoids of cystic fibrosis patients. Cell stem cell, 2013. 13(6): p. 653-658. 
35. Bakondi, B., et al., In vivo CRISPR/Cas9 gene editing corrects retinal dystrophy in the S334ter-3 rat model of autosomal dominant retinitis pigmentosa. Molecular Therapy, 2016. 24(3): p. 556563.

36. Ma, H., et al., Correction of a pathogenic gene mutation in human embryos. Nature, 2017. 548(7668): p. 413-419.

37. Peddle, C.F. and R.E. MacLaren, Focus: genome editing: the application of CRISPR/Cas9 for the treatment of retinal diseases. The Yale journal of biology and medicine, 2017. 90(4): p. 533.

38. Chang, C.-W., et al., Modeling human severe combined immunodeficiency and correction by CRISPR/Cas9-enhanced gene targeting. Cell reports, 2015. 12(10): p. 1668-1677.

39. Yang, Y., et al., A dual AAV system enables the Cas9-mediated correction of a metabolic liver disease in newborn mice. Nature biotechnology, 2016. 34(3): p. 334-338.

40. Ma, S., et al., 8 cell replacement after gene editing of a neonatal diabetes-causing mutation at the insulin locus. Stem cell reports, 2018. 11(6): p. 1407-1415.

41. Ye, L., et al., Seamless modification of wild-type induced pluripotent stem cells to the natural CCR5 32 mutation confers resistance to HIV infection. Proceedings of the National Academy of Sciences, 2014. 111(26): p. 9591-9596.

42. Li, C., et al., Inhibition of HIV-1 infection of primary CD4+ T-cells by gene editing of CCR5 using adenovirus-delivered CRISPR/Cas9. Journal of General Virology, 2015. 96(8): p. 2381-2393.

43. Wang, J. and S.R. Quake, RNA-guided endonuclease provides a therapeutic strategy to cure latent herpesviridae infection. Proceedings of the National Academy of Sciences, 2014. 111(36): p. 13157-13162.

44. Hu, Z., et al., Disruption of HPV16-E7 by CRISPR/Cas system induces apoptosis and growth inhibition in HPV16 positive human cervical cancer cells. BioMed research international, 2014. 2014.

45. Kennedy, E.M., et al., Inactivation of the human papillomavirus E6 or E7 gene in cervical carcinoma cells by using a bacterial CRISPR/Cas RNA-guided endonuclease. Journal of virology, 2014. 88(20): p. 11965-11972.

46. Yu, L., et al., Disruption of human papillomavirus 16 E6 gene by clustered regularly interspaced short palindromic repeat/Cas system in human cervical cancer cells. OncoTargets and therapy, 2015. 8: p. 37.

47. Dong, C., et al., Targeting hepatitis B virus cCCDNA by CRISPR/Cas9 nuclease efficiently inhibits viral replication. Antiviral research, 2015. 118: p. 110-117.

48. Gilbert, L.A., et al., CRISPR-mediated modular RNA-guided regulation of transcription in eukaryotes. Cell, 2013. 154(2): p. 442-451.

49. Qi, L.S., et al., Repurposing CRISPR as an RNA-guided platform for sequence-specific control of gene expression. Cell, 2013. 152(5): p. 1173-1183.

50. Kearns, N.A., et al., Functional annotation of native enhancers with a Cas9-histone demethylase fusion. Nature methods, 2015. 12(5): p. 401-403.

51. Thakore, P.I., et al., Highly specific epigenome editing by CRISPR-Cas9 repressors for silencing of distal regulatory elements. Nature methods, 2015. 12(12): p. 1143-1149.

52. Hilton, I.B., et al., Epigenome editing by a CRISPR-Cas9-based acetyltransferase activates genes from promoters and enhancers. Nature biotechnology, 2015. 33(5): p. 510-517.

53. Chen, B., et al., Dynamic imaging of genomic loci in living human cells by an optimized CRISPR/Cas system. Cell, 2013. 155(7): p. 1479-1491.

54. Anton, T., et al., Visualization of specific DNA sequences in living mouse embryonic stem cells with a programmable fluorescent CRISPR/Cas system. Nucleus, 2014. 5(2): p. 163-172.

55. Tanenbaum, M.E., et al., A protein-tagging system for signal amplification in gene expression and fluorescence imaging. Cell, 2014. 159(3): p. 635-646. 
56. Ma, H., et al., Multicolor CRISPR labeling of chromosomal loci in human cells. Proceedings of the National Academy of Sciences, 2015. 112(10): p. 3002-3007.

57. Deng, W., et al., CASFISH: CRISPR/Cas9-mediated in situ labeling of genomic loci in fixed cells. Proceedings of the National Academy of Sciences, 2015. 112(38): p. 11870-11875.

58. Zheng, A., et al., CRISPR/Cas9 genome-wide screening identifies KEAP1 as a sorafenib, lenvatinib, and regorafenib sensitivity gene in hepatocellular carcinoma. Oncotarget, 2019. 10(66): p. 7058.

59. Crowther, M.D., et al., Genome-wide CRISPR-Cas9 screening reveals ubiquitous $T$ cell cancer targeting via the monomorphic MHC class I-related protein MR1. Nature immunology, 2020. 21(2): p. 178-185.

60. Liu, Y., et al., Genome-wide screening for functional long noncoding RNAs in human cells by Cas9 targeting of splice sites. Nature Biotechnology, 2018. 36(12): p. 1203-1210.

61. Jason, S. and K. Yusa, Genome-wide CRISPR-Cas9 screening in mammalian cells. Methods, 2019. 164: p. 29-35.

62. Sharma, S. and E. Petsalaki, Application of CRISPR-Cas9 based genome-wide screening approaches to study cellular signalling mechanisms. International journal of molecular sciences, 2018. 19(4): p. 933.

63. Li, M., et al., Reassessment of the four yield-related genes Gn1a, DEP1, GS3, and IPA1 in rice using a CRISPR/Cas9 system. Frontiers in plant science, 2016. 7: p. 377.

64. Xu, R., et al., Rapid improvement of grain weight via highly efficient CRISPR/Cas9-mediated multiplex genome editing in rice. Journal of Genetics and Genomics= Yi chuan xue bao, 2016. 43(8): p. 529-532.

65. Li, X., et al., High-efficiency breeding of early-maturing rice cultivars via CRISPR/Cas9-mediated genome editing. Journal of genetics and genomics= Yi chuan xue bao, 2017. 44(3): p. 175-178.

66. Ma, L., et al., Essential role of sugar transporter OsSWEET11 during the early stage of rice grain filling. Plant and Cell Physiology, 2017. 58(5): p. 863-873.

67. Ueta, R., et al., Rapid breeding of parthenocarpic tomato plants using CRISPR/Cas9. Scientific reports, 2017. 7(1): p. 1-8.

68. Soyk, S., et al., Variation in the flowering gene SELF PRUNING 5 G promotes day-neutrality and early yield in tomato. Nature Genetics, 2017. 49(1): p. 162-168.

69. Jiang, W.Z., et al., Significant enhancement of fatty acid composition in seeds of the allohexaploid, Camelina sativa, using CRISPR/Cas9 gene editing. Plant biotechnology journal, 2017. 15(5): p. 648-657.

70. Sun, Y., et al., Generation of high-amylose rice through CRISPR/Cas9-mediated targeted mutagenesis of starch branching enzymes. Frontiers in plant science, 2017. 8: p. 298.

71. Qi, W., et al., High-efficiency CRISPR/Cas9 multiplex gene editing using the glycine tRNAprocessing system-based strategy in maize. BMC biotechnology, 2016. 16(1): p. 1-8.

72. Peng, A., et al., Engineering canker-resistant plants through CRISPR/Cas9-targeted editing of the susceptibility gene CS LOB 1 promoter in citrus. Plant biotechnology journal, 2017. 15(12): p. 1509-1519.

73. Wang, F., et al., Enhanced rice blast resistance by CRISPR/Cas9-targeted mutagenesis of the ERF transcription factor gene OsERF922. PloS one, 2016. 11(4): p. e0154027.

74. Nekrasov, V., et al., Rapid generation of a transgene-free powdery mildew resistant tomato by genome deletion. Scientific reports, 2017. 7(1): p. 1-6.

75. Pyott, D.E., E. Sheehan, and A. Molnar, Engineering of CRISPR/Cas9-mediated potyvirus resistance in transgene-free Arabidopsis plants. Molecular plant pathology, 2016. 17(8): p. 12761288.

76. Chandrasekaran, J., et al., Development of broad virus resistance in non-transgenic cucumber using CRISPR/Cas9 technology. Molecular plant pathology, 2016. 17(7): p. 1140-1153. 